\title{
Proposed experimental tests of the Bell-Kochen-Specker theorem*
}

\author{
Adán Cabello ${ }^{\dagger}$ \\ Departamento de Física Aplicada, \\ Universidad de Sevilla, 41012 Sevilla, Spain. \\ Guillermo García-Alcaine ${ }^{\ddagger}$ \\ Departamento de Física Teórica, \\ Universidad Complutense, 28040 Madrid, Spain.
}

August 1, 2018

\begin{abstract}
For a two-particle two-state system, sets of compatible propositions exist for which quantum mechanics and noncontextual hidden-variable theories make conflicting predictions for every individual system whatever its quantum state. This permits a simple all-or-nothing stateindependent experimental verification of the Bell-Kochen-Specker theorem.
\end{abstract}

PACS numbers: 03.65.Bz

\footnotetext{
${ }^{*}$ Phys. Rev. Lett. 80, 1797 (1998).

$\dagger$ Electronic address: fite1z1@sis.ucm.es

¥Electronic address: fite114@sis.ucm.es
} 
There are two main theorems on the impossibility of hidden variables in quantum mechanics (QM). The most general is the Bell-Kochen-Specker (BKS) theorem [1, 2] which excludes noncontextual hidden-variable (NCHV) theories (i. e., those in which the values of the physical observables are the same whatever the experimental context in which they appear). The other is Bell's theorem [3] which discards local hidden variables of the kind considered by Einstein, Podolsky, and Rosen [4]. Both theorems are mathematical statements which, as such, do not require any real experiment to be proved or disproved. Only if we want to investigate how nature behaves do we require actual experiments. There is a wide range of experiments which show that nature violates Bell's inequalities [5]. However, no empirical disproof of NCHV theories has yet been exhibited [6]. This situation can be explained by comparing the proofs of both theorems. Bell's inequalities [3] involve statistical magnitudes which can be calculated from measurements carried out in different subensembles of pairs. In contrast, the proofs of the BKS theorem [1, 2, 7] refer to a single individual system but involve noncompatible observables that cannot be measured in the same individual system. On the other hand, while Bell's inequalities are verified by any local hidden-variable theory independently of any QM assumptions, the proofs of the BKS theorem refer to NCHV theories that share some properties with QM. In this sense, the proofs of the BKS theorem are not entirely independent of the formal structure of QM. For these reasons, one could think that "the whole notion of an experimental test of [B]KS misses the point" [8].

In this paper we will show a situation, the first to our knowledge, in which NCHV theories, without any call to the formal structure of QM, make conflicting predictions with those of QM for every individual system and whatever its quantum state. These predictions can be tested by a joint measurement of one set of compatible propositions.

We propose the following situation. Consider an individual system of two spin- $\frac{1}{2}$ particles (or any other two-particle two-state system) initially prepared in an unspecified state. Suppose that a NCHV theory can describe that system. Noncontextuality here will mean that this hidden-variable theory satisfies the following two assumptions:

(i) Any one-particle observable (for a two-state system) can be assumed to have a definite value. This is a reasonable assumption for any NCHV theory since Gleason's theorem [9] is not valid for systems described by a Hilbert space of dimension two, and since the possibility of NCHV for these 
systems was explicitly proved by Bell [1] and by Kochen and Specker [2]. In particular, we will assume that the observables $A:=\sigma_{z}^{(1)}, B:=\sigma_{z}^{(2)}, a:=\sigma_{x}^{(1)}$, and $b:=\sigma_{x}^{(2)}$ (the spin components in units of $\hbar / 2$ in the $z$ and $x$ directions for particles one and two) have predefined noncontextual values either +1 or -1 . We will denote these values as $v(A), v(B), v(a)$, and $v(b)$. Then, considering the values of these four observables, $2^{4}$ different "states" could exist (for instance, one possible "state" is $v(A)=-v(B)=-v(a)=v(b)=+1$ ).

(ii) The value of a two-particle observable which is a product of oneparticle observables such as $A B$ (or $A b$, etc) is

$$
v(A B):=v(A) v(B) \text {. }
$$

Note that $A$ and $B$ are not only compatible observables but refer to two different particles 10. Definition (11) is a consequence of noncontextuality since one particular way of measuring the observable $A B$ is by measuring separately $A$ and $B$ and multiplying their results; but, in a NCHV theory, $v(A B)$ must be the same whatever the experimental context in which it appears.

Now we will show some predictions derived from these two assumptions. For that purpose consider the following four propositions:

$$
\begin{gathered}
P_{1}:=" A B=1 \quad \text { and } a b=1 ", \\
P_{2}:=" A B=-1 \quad \text { and } a b=-1 ", \\
P_{3}:=" A b=1 \text { and } a B=1 ", \\
P_{4}:=" A b=-1 \quad \text { and } a B=-1 " .
\end{gathered}
$$

Proposition $P_{1}$ has the value 1 (true) if the two-particle observables $A B$ and $a b$ have values +1 , and the value 0 (false) otherwise, etc. In a NCHV theory, the $\left\{P_{i}\right\}$ have predefined values related, using assumption (ii), to those of $A$, $B$, $a$, and $b$. For instance, $v\left(P_{1}\right)=1$ if $v(A)=v(B)$ and $v(a)=v(b)$, and zero otherwise.

As can be easily seen from the study of all the possible states of this NCHV theory, some predictions can be made:

NCHV1.- The propositions $P_{1}, P_{2}, P_{3}, P_{4}$ are not mutually exclusive: Two of them can be simultaneously true [for instance, $v\left(P_{1}\right)=v\left(P_{3}\right)=1$ in the state $v(A)=v(B)=v(a)=v(b)=+1$ ]. 
NCHV2.- $P_{1}, P_{2}, P_{3}, P_{4}$ are not exhaustive: All of them can be simultaneously false [for instance, $v\left(P_{1}\right)=v\left(P_{2}\right)=v\left(P_{3}\right)=v\left(P_{4}\right)=0$ in the state $v(A)=v(B)=v(a)=-v(b)=+1]$.

Indeed, checking all the possible states, NCHV1 and NCHV2 can be summarized as follows:

NCHV3.- In a NCHV theory, the values of $P_{1}, P_{2}, P_{3}$, and $P_{4}$ in a joint measurement would be either 4 zeros - all the propositions are false-, or 2 ones and 2 zeros -2 propositions are true and 2 are false-.

Note that the predictions NCHV1, NCHV2, and NCHV3 are entirely independent of the formal structure of QM.

What are the corresponding quantum predictions? First, let us see the quantum representatives of propositions $P_{1}, P_{2}, P_{3}$, and $P_{4}$. If $\hat{A}, \hat{B}, \hat{a}$, and $\hat{b}$ denote the self-adjoint operators representing the observables $A, B, a$, and $b$, the proposition $P_{i}$ is represented by the projector $\hat{P}_{i}:=\left|\psi_{i}\right\rangle\left\langle\psi_{i}\right|$, where $\left\{\left|\psi_{i}\right\rangle\right\}$ are the states defined by the following eigenvalue equations [11:

$$
\begin{gathered}
\hat{A} \otimes \hat{B}\left|\psi_{1}\right\rangle=\left|\psi_{1}\right\rangle, \quad \hat{a} \otimes \hat{b}\left|\psi_{1}\right\rangle=\left|\psi_{1}\right\rangle, \\
\hat{A} \otimes \hat{B}\left|\psi_{2}\right\rangle=-\left|\psi_{2}\right\rangle, \quad \hat{a} \otimes \hat{b}\left|\psi_{2}\right\rangle=-\left|\psi_{2}\right\rangle, \\
\hat{A} \otimes \hat{b}\left|\psi_{3}\right\rangle=\left|\psi_{3}\right\rangle, \quad \hat{a} \otimes \hat{B}\left|\psi_{3}\right\rangle=\left|\psi_{3}\right\rangle, \\
\hat{A} \otimes \hat{b}\left|\psi_{4}\right\rangle=-\left|\psi_{4}\right\rangle, \quad \hat{a} \otimes \hat{B}\left|\psi_{4}\right\rangle=-\left|\psi_{4}\right\rangle .
\end{gathered}
$$

As can be easily seen, the projectors $\hat{P}_{1}, \hat{P}_{2}, \hat{P}_{3}$, and $\hat{P}_{4}$ are mutually orthogonal,

$$
\hat{P}_{i} \hat{P}_{j}=0 \quad \text { if } \quad i \neq j .
$$

Therefore, according to QM:

QM1.-The propositions $P_{1}, P_{2}, P_{3}$, and $P_{4}$ are mutually exclusive: Two of them cannot be simultaneously true.

Moreover, it can be checked that the projectors $\hat{P}_{1}, \hat{P}_{2}, \hat{P}_{3}$, and $\hat{P}_{4}$ form a resolution of the identity, i.e.,

$$
\hat{P}_{1}+\hat{P}_{2}+\hat{P}_{3}+\hat{P}_{4}=\hat{1} .
$$

Therefore, according to QM:

QM2.- $P_{1}, P_{2}, P_{3}$, and $P_{4}$ are exhaustive: Not all of them can be simultaneously false. 
Indeed, from the mathematical properties (10) and (11) follows a third physical prediction which includes QM1 and QM2:

QM3.-According to QM, in any joint measurement of $P_{1}, P_{2}, P_{3}$, and $P_{4}$ in the same individual system, one and only one of the propositions will be true and the other three will be false, whatever the preparation of the state.

Clearly, NCHVi and QMi are conflicting physical predictions. The situation at this point is similar to that which appears between Bell's inequalities and QM: We have two theories with contradictory predictions. Now we have to propose an experiment to check how nature behaves.

How could a joint measurement of $P_{1}, P_{2}, P_{3}$, and $P_{4}$ be possible? Until now we have assumed that the propositions $P_{1}, P_{2}, P_{3}$, and $P_{4}$ are compatible. This remains to be justified. Of course, we have seen that the projectors $\hat{P}_{1}, \hat{P}_{2}, \hat{P}_{3}$, and $\hat{P}_{4}$ commute, and it is a generally accepted assumption of QM that commuting operators correspond to compatible observables. The reason for this assumption is that, if there is a set of pairwise commuting selfadjoint operators, then there exists a nontrivial maximal-nondegenerateoperator $\hat{H}$ commuting with all $\hat{P}_{i}$, such that $\hat{P}_{i}=f_{i}(\hat{H})$ [12]. However, this justification hinges on the existence of a physical observable $H$ which corresponds to the operator $\hat{H}$. In our case, such operator can be

$$
\hat{H}=\sum_{i=1}^{4} c_{i} \hat{P}_{i},
$$

where the $\left\{c_{i}\right\}$ are arbitrary distinct real numbers. Then, it is easily checked that

$$
\hat{P}_{i}=\prod_{j \neq i} \frac{\hat{H}-c_{j} \hat{1}}{c_{i}-c_{j}} .
$$

Optical observables corresponding to operators of the form (12) for twoparticle systems have been proposed and actual experimental results are expected to be presented soon [13]. On the other hand, the proposals [14 for experiments designed to measure the Bell operator 15 used for quantum teleportation [16] can be modified to measure operators of the form (12) [17].

In summary, we have showed that there are situations in nature in which NCHV theories, without any call to the formal structure of QM, make conflicting predictions with those of QM for every individual system whatever its quantum state. An experimental test of these predictions requires the measurement of a particular set of compatible propositions. Optical versions 
of experiments related with these propositions have been proposed for other purposes, and actual experimental results based on these proposals are expected to be presented soon.

The authors wish to thank Asher Peres for his comments and suggestions, and David Mermin for his observations and criticisms; both have been essential in the writing of this Letter. We also acknowledge comments by Ignacio Cirac and Emilio Santos. One of us (A. C.) thanks Harvey Brown, Gonzalo García de Polavieja, and Erik Sjöqvist for useful discussions, and for their hospitality at Oxford. 


\section{References}

[1] J. S. Bell, Rev. Mod. Phys. 38, 447 (1966).

[2] S. Kochen and E. P. Specker, J. Math. Mech. 17, 59 (1967).

[3] J. S. Bell, Physics 1, 195 (1964).

[4] A. Einstein, B. Podolsky, and N. Rosen, Phys. Rev. 47, 777 (1935).

[5] A. Aspect, J. Dalibard, and G. Roger, Phys. Rev. Lett. 49, 1804 (1982); Y. H. Shih and C. O. Alley, Phys. Rev. Lett. 61, 2921 (1988); J. G. Rarity and P. R. Tapster, Phys. Rev. Lett. 64, 2495 (1990); P. G. Kwiat, K. Mattle, H. Weinfurter, A. Zeilinger, A. V. Sergienko, and Y. H. Shih, Phys. Rev. Lett. 75, 4337 (1995).

[6] E. Santos, in Quantum Mechanics Versus Local Realism: The EinsteinPodolsky-Rosen Paradox, edited by F. Selleri (Plenum, New York, 1988).

[7] N. D. Mermin, Phys. Rev. Lett. 65, 3373 (1990); Rev. Mod. Phys. 65, 803 (1993); A. Peres, Phys. Lett. A 151, 107 (1990); J. Phys. A 24, L175 (1991); Quantum Theory: Concepts and Methods (Kluwer, Dordrecht, 1993); M. Kernaghan, J. Phys. A 27, L829 (1994); M. Kernaghan and A. Peres, Phys. Lett. A 198, 1 (1995); A. Cabello and G. García Alcaine, J. Phys. A 29, 1025 (1996); A. Cabello, J. M. Estebaranz, and G. García Alcaine, Phys. Lett. A 212, 183 (1996); Phys. Lett. A 218, 115 (1996).

[8] N. D. Mermin (private communication).

[9] A. M. Gleason, J. Math. Mech. 6, 885 (1957).

[10] Definition (1) is a particular case of the product rule. See, for instance, A. Fine and P. Teller, Found. Phys. 8, 629 (1978).

[11] We can write down explicitly these states on the basis of common eigenstates of $\sigma_{z}^{(1)}$ and $\sigma_{z}^{(2)}:\left|\psi_{1}\right\rangle=\frac{1}{\sqrt{2}}(|++\rangle+|--\rangle),\left|\psi_{2}\right\rangle=$ $\frac{1}{\sqrt{2}}(|+-\rangle-|-+\rangle), \quad\left|\psi_{3}\right\rangle=\frac{1}{2}(|++\rangle+|+-\rangle+|-+\rangle-|--\rangle),\left|\psi_{4}\right\rangle=$ $\frac{1}{2}(-|++\rangle+|+-\rangle+|-+\rangle+|--\rangle)$.

[12] J. von Neumann, Ann. of Math. 32, 191 (1931). 
[13] M. Zukowski, A. Zeilinger, and M. A. Horne, Phys. Rev. A 55, 2564 (1997).

[14] J. I. Cirac and A. S. Parkins, Phys. Rev. A 50, R4441 (1994); S. L. Braunstein and A. Mann, Phys. Rev. A 51, R1727 (1995); Phys. Rev. A 53, 630 (1996); M. H. Y. Moussa, Phys. Lett. A 231, 23 (1997).

[15] S. L. Braunstein, A. Mann, and M. Revzen, Phys. Rev. Lett. 68, 3259 (1992).

[16] C. H. Bennett, G. Brassard, C. Crépeau, R. Jozsa, A. Peres, and W. Wootters, Phys. Rev. Lett. 70, 1895 (1993).

[17] Indeed, the states $\left|\psi_{i}\right\rangle$ appearing in Eqs. (6)-(9) are almost the same as those of the Bell operator basis, $\left|\Phi^{ \pm}\right\rangle$and $\left|\Psi^{ \pm}\right\rangle$(Ref. [15]), namely $\left|\psi_{1}\right\rangle=\left|\Phi^{+}\right\rangle,\left|\psi_{2}\right\rangle=\left|\Psi^{-}\right\rangle,\left|\psi_{3}\right\rangle=\frac{1}{\sqrt{2}}\left(\left|\Psi^{+}\right\rangle+\left|\Phi^{-}\right\rangle\right)$, and $\left|\psi_{4}\right\rangle=$ $\frac{1}{\sqrt{2}}\left(\left|\Psi^{+}\right\rangle-\left|\Phi^{-}\right\rangle\right)$. 